\title{
Computed Tomography of the Chest with
} Contrast

National Cancer Institute

\section{Source}

National Cancer Institute. Computed Tomography of the Chest with Contrast. NCI

Thesaurus. Code C137883.

Computed tomography of the chest with the use of a contrast agent to enhance the image. 